Trauma Berufskrankh 2016 · [Suppl 3]: 18:S270-S276

DOI 10.1007/s10039-015-0101-x

Online publiziert: 26. November 2015

๑) Springer-Verlag Berlin Heidelberg 2015

CrossMark

\author{
M.S.T. Wähmann ${ }^{1} \cdot$ M. Rahimi ${ }^{1}$ - A. Daigeler ${ }^{2}$ B. Bickert ${ }^{1} \cdot$ T. Kremer $^{1} \cdot$ U. Kneser ${ }^{1}$ \\ ${ }^{1}$ Klinik für Hand-, Plastische- und Rekonstruktive Chirurgie - Schwerbrandverletztenzentrum, \\ BG Klinik Ludwigshafen, Lehrkrankenhaus der Ruprecht-Karls-Universität Heidelberg, \\ Ludwigshafen, Deutschland \\ 2 Universitätsklinik für Plastische Chirurgie und Schwerbrandverletzte, Handchirurgiezentrum, \\ Operatives Referenzzentrum für Gliedmaßentumoren, Berufsgenossenschaftliches \\ Universitätsklinikum Bergmannsheil Bochum, Bochum, Deutschland
}

\title{
Nekrotisierende Fasziitis der oberen Extremität
}

\section{Diagnostik und Therapiealgorithmus}

Die nekrotisierende Fasziitis (NF) ist die pathologisch deskriptive Bezeichnung für eine häufig fulminant verlaufende bakterielle Weichteilinfektion, die mit Fasziennekrosen, Nekrosen des subkutanen Fettgewebes sowie der darüber liegenden Haut einhergeht $[8,14]$. In der Geschichte wurde sie zum ersten Mal während des amerikanischen Bürgerkriegs registriert und von dem Militärchirurgen Joseph Jones [13] im Jahre 1871 zunächst als „Krankenhausgangrän“ beschrieben. Fournier [6] postulierte 1883 eine Sonderform der NF mit spezifischem Befall des Perineums und der Genitalien. Der Begriff „nekrotisierende Fasziitis“ wurde erstmalig 1952 von Wilson [32] geprägt, indem dieser eine durch Streptokokken induzierte Fasziennekrose pathognomonisch nachweisen konnte. Zwar handelt es sich mit 1,5-3,5 Fällen pro 100.000 Einwohner $[5,18]$ um ein seltenes Krankheitsbild, jedoch zeigen aktuelle Studien eine zunehmende Inzidenz [14, 23]. In den USA werden jährlich ca. 500-1000 Neuerkrankungen registriert [31]. Männer sind allgemein häufiger als Frauen betroffen [4]. Trotz Optimierung interdisziplinärer Therapiealgorithmen liegen die Mortalitätsraten heute zwischen $15 \%$ und $46 \%$ [20]. Risikofaktoren für das Auftreten einer NF sind u. a. Diabetes mellitus, chronische Niereninsuffizienz, Adipositas, intravenöser Drogenabusus, aber auch die Einnahme von Immunsuppressiva oder ein gestörtes Immunsystem $[3,25,27]$. In einer Studie wurde gezeigt, dass an NF verstorbene Patienten mit durchschnittlich 3,6 Nebenerkrankungen eine signifikant höhere Anzahl von Begleiterkrankungen aufwiesen als die überlebenden Patienten mit durchschnittlich 1,6 Nebenerkrankungen [24]. Bereits bei klinischer Verdachtsdiagnose besteht die Indikation zur sofortigen operativen Therapie [1].

\section{Ätiologie und Klassifikation}

Die Entstehung dieser Weichgewebeinfektion ist bis heute nicht vollständig geklärt. Die bakterielle Eintrittspforte als Auslöser variiert erheblich und kann von kleinen Bagatellverletzungen bis zu ausgedehnten Weichteiltraumata reichen. Insbesondere bei Befall der oberen Extremität gingen häufig Insektenstiche, intravenöser Drogenabusus, kutane Abszesse, kleinere Traumata oder Bissverletzungen voraus $[7,28]$. Über Hautläsionen und Wunden gelangen die Krankheitserreger in das subkutane Gewebe, um sich in der Tiefe entlang der Faszien und Septen weiter zu verbreiten. Charakteristisch sind eine große Dynamik der Ausbreitung mit frühzeitiger Beteiligung des Subkutangewebes, entzündliche Umgebungsreaktionen und septisch-toxische Effekte durch Einbeziehung des Gefäßsystems. Ähnlich heterogen und breit gefächert wie die Eintrittspforte ist das mikrobiologische Keimspektrum, in dem eine Vielzahl an bakteriellen Erregern als potenzielle
Ursache infrage kommt. Am häufigsten konnten Streptokokkenstämme nachgewiesen werden. Oftmals zeigen sich auch polymikrobielle Infektionen mit diversen Staphylokokkenarten in Kombination mit $\beta$-hämolysierenden Streptokokken. Ebenso wurden zahlreiche Enterobacter-Spezies, Klebsiella, Proteus mirabilis, Pseudomonaden oder auch anaerobe grampositive Kokken- und Clostridienarten isoliert [3]. Die aktuell etablierte Klassifikation unterscheidet die NF anhand des ursächlichen Keimspektrums (• Tab. 1). Bei Typ I handelt es sich um eine polymikrobielle Infektion. Bei Typ II liegt zumeist eine Monoinfektion vor und Typ III wird durch eine Besiedelung von Clostridien verursacht [17]. Lassen sich Pilze als Krankheitserreger diagnostizieren, liegt Typ IV vor [3]. Lediglich bei Typ I und III kommt es zu einer Gasbildung.

\section{Diagnostik}

\section{Klinische Symptomatik}

Eine frühzeitige Diagnostik ist aufgrund der rasanten Krankheitsprogredienz von großer Bedeutung, um durch sofortige Einleitung der chirurgischen Therapie der hohen Letalität entgegenzuwirken. Jedoch gestaltet sich die Diagnosestellung, die zunächst anhand klinischer Zeichen erfolgt, im frühen Anfangsstadium äußerst schwierig. Da sich die NF in tieferen Gewebeschichten fort- 


\begin{tabular}{|c|c|c|}
\hline & Klinische Symptomatik & Keimspektrum \\
\hline Typ I & $\begin{array}{l}\text { Fett- und Fasziennekrosen, teilweise mit } \\
\text { Gasbildung }\end{array}$ & Aerob-anaerobe Mischinfektion \\
\hline Typ II & $\begin{array}{l}\text { Ausgeprägte Nekrosen mit schichtüber- } \\
\text { greifender Beteiligung ohne Gasbildung }\end{array}$ & $\begin{array}{l}\text { Streptokokken der Gruppe A mit oder ohne } \\
\text { eine simultane Infektion mit Staphylococcus } \\
\text { aureus/epidermidis, meistens Monoinfektion }\end{array}$ \\
\hline Typ III & $\begin{array}{l}\text { Fulminante Myonekrosen mit Gas- } \\
\text { bildung }\end{array}$ & $\begin{array}{l}\text { Gramnegative Bakterien, häufig meerwasser- } \\
\text { assoziierte Mikroorgansimen }\end{array}$ \\
\hline Typ IV & $\begin{array}{l}\text { Unterschiedlich ausgeprägte Nekrosen- } \\
\text { bildung möglich, keine Gasbildung }\end{array}$ & Pilze (Candida, Aspergillus) \\
\hline
\end{tabular}

setzt, zeigen Hautveränderungen meist nicht das gesamte Ausmaß des Befalls oder sind initial nicht vorhanden. Die klinischen Symptome wie Erythem, Ödem oder erhöhte Temperaturen entsprechen einer lokalen oder systemischen Infektion. Vesikuläre Hauteffloreszenzen oder ein Crepitus sind eher selten. Im Anfangsstadium ist eine für den weiteren Therapieerfolg dringend erforderliche Unterscheidung zwischen einer NF und anderen Weichgewebeinfektionen sehr erschwert. Relevante Differenzialdiagnosen sind Erysipel, Zellulitis, Pyomyositis oder besonders im Bereich der Hände phlegmonöse Infekte. Diese gehen i.d.R. ebenfalls mit klinischen Symptomen wie Rötung, Schwellung oder Spannungsgefühl einher, sodass laut Studienlage bis zu $35 \%$ der NF als nichtnekrotisierende Infektion des Weichteilgewebes fehlinterpretiert wurden [10].

Charakteristisch ist im frühen Stadium ein unverhältnismäßig starker Schmerz ("pain out of proportion“), der durch die rasche Infektausbreitung mit Ischämie entlang der Faszien entsteht und nicht mit dem Ausmaß der Hautbeteiligung korreliert. Mit Voranschreiten der Infektion mit hämatogener Keiminfiltration zeigt sich das klinische Bild einer Sepsis mit Vigilanzminderung, Kreislaufdepression und Fieber [26, 30]. Begünstigend für die Entwicklung einer $\mathrm{NF}$ an der oberen Extremität sind anatomische Besonderheiten. So kann sich die Erkrankung über Bagatelltraumata an der Hand rasch entlang der Septen des Subkutangewebes, die senkrecht zu Knochen, Sehnenscheiden und Palmaraponeurose verlaufen, ausbreiten. Dabei ist die Abgrenzung zu weniger dramatischen Infektionen, wie z. B. Phlegmonen, nicht immer einfach.

\section{Radiologische Bildgebung}

Die radiologische Bildgebung ist bei der NF relativ zeitaufwendig und insbesondere im frühen Stadium können pathologische bildmorphologische Befunde gänzlich fehlen. Im Verdachtsfall hat die chirurgische Exploration stets die höchste Priorität und sollte keinesfalls durch eine radiologische Diagnostik verzögert werden. Im konventionellen Röntgenbild können neben einem verbreiterten Weichteilschatten als spezifisches Zeichen Gaseinschlüsse sichtbar sein. Jedoch ist das subkutane Emphysem nur in ca. einem Viertel der Fälle vorhanden, sodass fehlende Gasbildung eine NF nicht ausschließen kann [16]. Die Computertomographie (CT) und die Magnetresonanztomographie (MRT) liefern weiterführende Hinweise auf eine NF. Als charakteristische Zeichen lassen sich Gaseinschlüsse entlang der Faszien, Fettgewebsnekrosen sowie eine asymmetrische Faszienverdickung diagnostizieren. Dabei bietet die MRT durch eine detailliertere Darstellung von Weichteilveränderungen Vorteile. An das Vorliegen einer NF lassen Flüssigkeitsansammlungen der tiefen Faszien sowie Verdickung und erhöhte Signalintensität in der T2-Gewichtung denken.

Insgesamt ist die zeitaufwendigere MRT aufgrund ihrer hohen Sensitivität von $100 \%$ und Spezifität von $80 \%$ der CT bei der Diagnosestellung überlegen [25]. In der Praxis wird häufig aufgrund des Zeitfaktors zugunsten der chirurgischen Exploration auf eine ausführliche Bildgebung verzichtet. Als Verlaufskontrolle ist eine CT- oder MRT-Untersuchung geeignet, um eine Progression der Infektion auszuschließen und verbliebene Nekrosezonen zu detektieren.

\section{Mikrobiologie}

Im Rahmen des ersten chirurgischen Débridements ist eine Probenentnahme zur mikrobiologischen Aufarbeitung obligat, um nach der initialen Phase der kalkulierten Antibiotikagabe eine erregerspezifische Antibiotikatherapie einleiten zu können. Die Gewebeprobe hat gegenüber mikrobiologischen Abstrichen eine höhere Sensitivität und kann in ca. $90 \%$ der Fälle einen Keimnachweis liefern. Ergänzend sollten Blutkulturen bei septischen Patienten als Standard durchgeführt werden, um das gesamte Erregerspektrum zu erfassen. Bis zum Vorliegen eines mikrobiologischen Keimnachweises sowie korrelierenden Antibiogramms sollte eine kalkulierte Antibiotikatherapie ohne Zeitverzögerung eingeleitet werden. Nach Auswertungen der letzten Jahre handelt es sich häufiger um poly- als um monomikrobielle Infektionen [7].

\section{Histopathologie}

Die histopathologische Aufarbeitung der intraoperativ entnommenen Proben dient der Diagnosesicherung der NF. Das therapeutische Vorgehen wird durch die Histologie jedoch nicht beeinflusst. Im Frühstadium lässt sich eine epidermale Nekrose mit interzellulärem Ödem und Hämorrhagien feststellen [21]. Weitere histopathologische Charakteristika sind eine polymorphkernige Infiltration der Dermis, fibroblastische Proliferation, Mikrothromben sowie Kolliquationsnekrosen. In fortgeschrittenen Stadien kann eine vollständige Nekrose der Dermis, Subkutis und der Faszie beobachtet werden [18]. Erst im Spätstadium kommt es auch zu einem nekrotischen Zerfall der Muskulatur.

\section{Therapie}

\section{Operative Verfahren}

Im frühen Stadium einer NF bestehen meist unspezifische Symptome. Charakteristische Zeichen wie Nekrosenoder Blasenbildung sind bei über $50 \%$ der Patienten initial nicht vorhanden. Da die Prognose des Betroffenen insbesondere von der zeitnahen und 
Trauma Berufskrankh 2016 · [Suppl 3]: 18:S270-S276 DOI 10.1007/s10039-015-0101-x

(c) Springer-Verlag Berlin Heidelberg 2015

M.S.T. Wähmann · M. Rahimi · A. Daigeler · B. Bickert · T. Kremer · U. Kneser

Nekrotisierende Fasziitis der oberen Extremität. Diagnostik und Therapiealgorithmus

Zusammenfassung

Hintergrund. Die nekrotisierende Fasziitis

ist eine seltene bakterielle Infektion des sub-

kutanen Gewebes sowie der Faszien und bei verzögerter, inadäquater Therapie mit einer hohen Sterblichkeit assoziiert.

Pathogenese. Ursächlich für die Infektionsausbreitung sind meist Bagatelltraumata und Weichteilverletzungen, die als Eintrittspforte für die bakteriellen Krankheitserreger dienen. Häufig sind immunsupprimierte Patienten mit Risikofaktoren wie Diabetes mellitus, Niereninsuffizienz oder Adipositas betroffen. Diagnose. Für die Diagnosestellung sind die Anamnese des Patienten und die Klinik entscheidend. Anfangs bestehen unspezifische Symptome einer Entzündung wie
Rötung, Schwellung, Schmerzen oder Fieber. Charakteristisch ist ein rasches Voranschreiten der Erkrankung. Eine Diagnosesicherung erfolgt in erster Linie durch histopathologische und mikrobiologische Auswertung der intraoperativen Biopsien. Labordiagnostik und radiologische Bildgebung sind anfänglich oft unspezifisch.

Therapie. Bei einer nekrotisierenden Fasziitis ist die sofortige notfallmäßige Operation indiziert, um das rasche Voranschreiten dieser lebensbedrohlichen Infektionskrankheit zu verhindern. Prognostisch entscheidend ist ein radikales Débridement der infizierten Gewebeareale. In den meisten Fällen sind mehrere Operationen notwendig. Zur
Rekonstruktion der entstandenen Weichteildefekte sind je nach Defektgröße und -tiefe Spalthauttransplantationen sowie gestielte oder freie Lappenplastiken erforderlich. In der Infektsituation ist eine breite kalkulierte Antibiotikatherapie (z. B. Tripleantibiose mit Penicillin, Clindamycin, Meronem) obligat. Bei ausgedehnten Befunden sollte der Patient ggf. in ein Schwerbrandverletztenzentrum zur Oberflächentherapie verlegt werden.

\section{Schlüsselwörter}

Muskuloskeletale Erkrankungen .

Débridement $\cdot$ Antibiotika $\cdot$ Intensivstation . Weichteilverletzungen

\section{Necrotizing fasciitis of the upper extremities. Diagnostics and therapy algorithm}

\section{Abstract}

Background. Necrotizing fasciitis is a rare bacterial infection of the subcutaneous tissue and the fascia, which is associated with a high mortality rate in the case of delayed and inadequate therapy.

Pathogenesis. The infection spreading along the fascia is usually caused by minor trauma or soft tissue injuries. Tiny erosions of the skin are sufficient as an entry portal for the pathogenic bacteria. Immunosuppressed patients with risk factors such as diabetes mellitus, renal failure and obesity are frequently affected by this disease.

Diagnosis. The diagnosis is established based on the patient medical history and the clinical findings. Initially unspecific symptoms of inflammation are present, such as local redness, swelling, pain and fever. Necrotizing fasciitis is characterized by a rapid progression with blistering, severe pain and systemic effects of sepsis with circulatory depression and depressed consciousness. A diagnosis is confirmed by histopathological and microbiological evaluation of biopsiy samples taken during surgical exploration. Laboratory diagnostics and radiological imaging are often unspecific in the initial phase.

Therapy. Necrotizing fasciitis is considered to be a life-threatening condition necessitating immediate surgical treatment to prevent rapid progress. Radical debridement of all affected tissues is of paramount importance with respect to overall survival and functional outcome. In most cases several operations are needed to control the infection. Defect reconstruction requires an individualized surgical strategy and can be achieved using split skin grafts and pedicled or free flaps depending on the defect size and depth. In the acute situation a calculated broad spectrum antibiotic therapy is required (e.g. triple therapy using penicillin, clindamycin and meropenem). In cases with extensive soft tissue defects transfer of the patient to a burn center is indicated for critical care and optimized body surface treament.

\section{Keywords}

Musculoskeletal diseases - Debridement . Antibiotics · Intensive care unit · Soft tissue injuries adäquaten chirurgischen Therapie abhängt, muss bereits beim Verdacht auf eine NF die Indikation zur notfallmäßigen chirurgischen Exploration gestellt werden ([11]; - Abb. 1). Die Letalitätsrate steigt durch einen verzögerten Therapiebeginn. In der Literatur wurde bei chirurgischen Eingriffen, die $24 \mathrm{~h}$ nach Aufnahme in der Klinik zeitverzögert durchgeführt wurden, eine 9-fach erhöhte Letalität beschrieben [33].

Das Ziel des chirurgischen Débridements ist die vollständige Exzision des erkrankten Gewebes mit besonderem Augenmerk auf die betroffenen
Faszien, die gräulich-nekrotisch verändert und nicht mehr an der Muskulatur adhärieren. Ein charakteristischer Befund ist zudem milchig-trübe Flüssigkeit, die als „Abwaschwasser-Pus“ („dish-water pus“) bezeichnet wird. Nur wenn ein radikales Débridement mit vollständiger Entfernung aller Nekrosen und ohne Bedenken vor Schaffung großer Weichteildefekte erfolgt, kann ein Überleben des Patienten erreicht werden. Aus dieser Tatsache geht das ungeschriebene Gesetz "Be bloody, bold, and resolute" hervor [9]. Im Bereich der distalen oberen Extremität führt ein adäquates, radikales
Débridement aufgrund des geringen Muskelmantels häufig zu ausgedehnten Defekten mit Exposition von Sehnen, Knochen, Nerven oder Blutgefäßen, die im Intervall mit Lappenplastiken rekonstruiert werden müssen. Die Entscheidung zu einem Débridement mit derartig weitreichenden Konsequenzen erfordert große klinische Erfahrung. Ist der Befund bereits fortgeschritten, kann bei der NF der oberen Extremität sogar primär eine Amputation von Fingern oder auch in extremen Ausnahmefällen der ganzen Hand indiziert sein, wenn nur so ein Ausbreiten auf den Körper- 


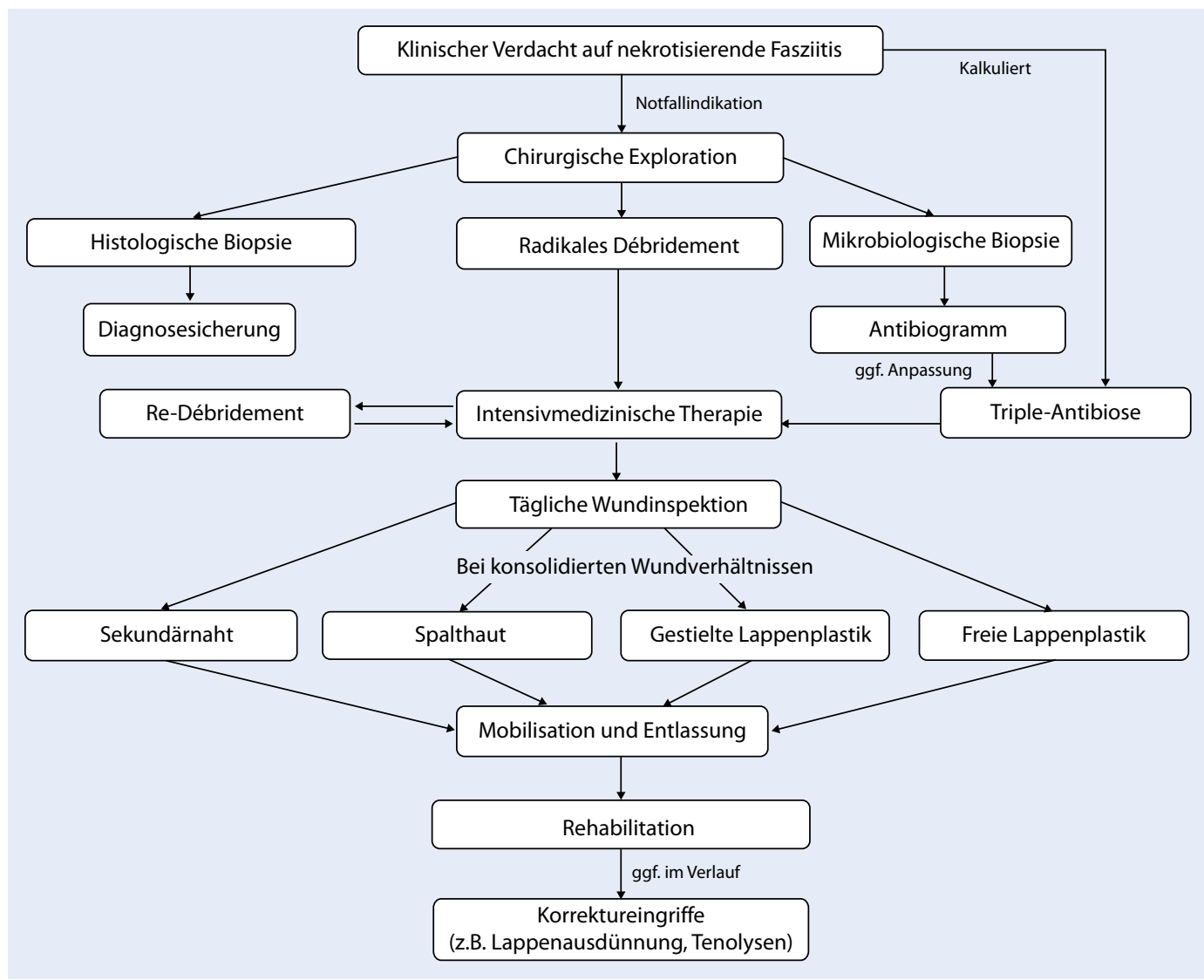

Abb. $1<$ Therapieschema der nekrotisierenden Fasziitis

stamm sicher verhindert und das Überleben des Patienten gewährleistet werden kann [12]. Hier gilt dann klar das Konzept „life before limb“. Bei vitaler Bedrohung führt diese mitunter radikale chirurgische Therapie zu einer deutlich niedrigeren Mortalität bei isoliertem Extremitätenbefall im Vergleich zu Manifestationen anderer Körperregionen [20]. Allerdings muss festgestellt werden, dass die primäre Amputation infolge einer NF der oberen Extremität eine höchst seltene Indikation darstellt [19, 29].

Ein primärer Wundverschluss nach dem Débridement ist i.d.R. keine Therapieoption, sodass als Standardverfahren die offene Wundbehandlung mit antiseptikagetränkten Bauchtüchern empfohlen werden kann. Theoretisch stellt auch die Anlage von Unterdruckverbandssystemen eine Alternative dar. Im eigenen Patientengut kam es aber unter dieser Therapie gelegentlich zum fulminanten Aufflammen der Infektion mit septischen Verläufen, sodass wir derzeit Unterdruckverbände nur selten und erst nach sicherer Sanierung des Infekts einsetzen.
Im Allgemeinen erfolgt innerhalb der ersten 48 h nach dem Erstdébridement eine geplante Second-Look-Operation. Nach eigener klinischer Erfahrung sind abhängig vom klinischen Verlauf und der Ausdehnung durchschnittlich 3 bis maximal 6 Revisionseingriffe bis zur kompletten lokalen Sanierung des Infektherds notwendig. Insbesondere an der Hand sollte der Verlust von funktionell relevantem Gewebe sorgfältig gegen die Notwendigkeit eines adäquaten Débridements abgewogen werden, sodass u. U. auch ein recht kurzfristiges zweites Débridement erforderlich werden kann. Dessen Zeitpunkt orientiert sich an dem klinischen Befund im Rahmen der täglichen Verbandswechsel, den Infektwerten in der engmaschigen Labordiagnostik sowie dem VitalparameterMonitoring auf der Intensivstation. In der initialen Phase rechtfertigt schon eine geringfügige Verschlechterung des klinischen Zustands die erneute operative Revision, da es mitunter trotz radikalen Débridements und kalkulierter Antibiotikatherapie zum Progress der NF kommen kann.

\section{Intensivmedizinische Behandlung}

Die Behandlung einer ausgedehnten NF der oberen Extremität sollte idealerweise in einem Zentrum mit handchirurgischer und rekonstruktiver Expertise sowie mit Überwachungsmöglichkeit auf einer chirurgischen Intensivstation stattfinden. Aufgrund der aufwendigen Oberflächentherapie sind insbesondere auf Intensivstationen für Schwerbrandverletzte optimale Bedingungen gegeben. Hier kann bei den oft hämodynamisch instabilen, septischen Patienten eine adäquate Volumentherapie leitliniengerecht eingeleitet werden. Die kardiopulmonale Überwachung sollte unter kontinuierlichem Monitoring erfolgen $[2,15,22]$. Zudem sollte das betreuende Team auf der Intensivstation mit ausgedehnten Verbandswechseln und Beurteilung des Oberflächenbefunds vertraut sein.

\section{Antibiotika}

Jeder Patient sollte bei klinischem Verdacht auf eine NF eine kalkulierte 

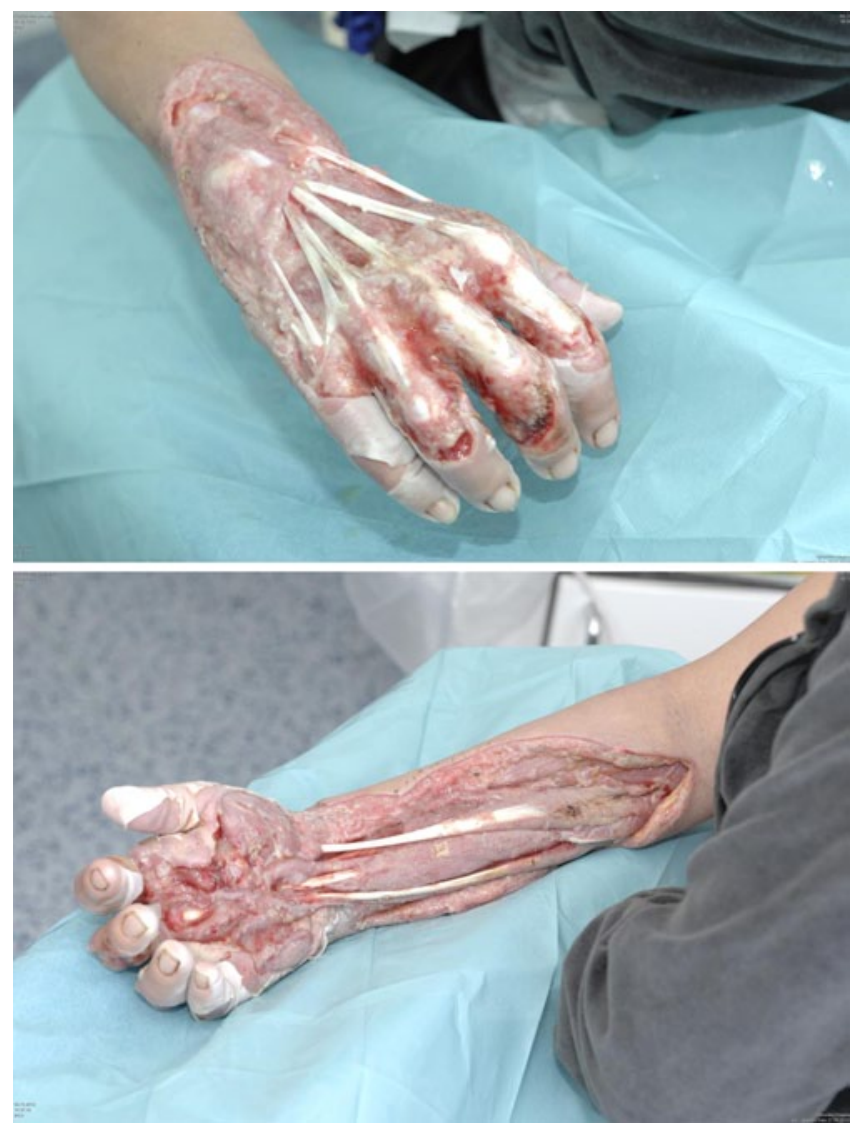

Abb. $2 \varangle$ Klinischer Befund nach mehrfachem Débridement streck- und beugeseitig am Unterarm sowie an der Hand

breite Antibiotikatherapie erhalten. Die Kombination aus 3 Antibiotika unterschiedlicher Wirkstoffklassen, z. B. Penicillin G (4-mal 2,5 Mio. E/Tag i.v.), Clindamycin (3-mal $900 \mathrm{mg} / \mathrm{Tag}$ i.v.) und Meropenem (3-mal $1 \mathrm{~g} / \mathrm{Tag}$ i.v.), gilt derzeit als etabliert und hat sich im eigenen Patientengut bewährt [17]. Häufig nachgewiesene $\beta$-hämolysierende Streptokokken der Gruppe A sind ausnahmslos gegen Penicillin und Cephalosporine sensitiv. Penicillin wirkt insgesamt gegen grampositive Kokken und Clindamycin gegen anaerobe Keime. Letztgenanntes vermindert über die Hemmung der Proteinsynthese auch die Produktion des Streptokokkentoxins sowie des MProteins. Bei Verdacht auf eine Infektion mit methicillinresistentem Staphylococcus aureus (MRSA) kann das Regime ggf. um Vancomycin ergänzt werden [2]. Nach Vorliegen des Antibiogramms sollte die Sensitivität überprüft und die Therapie angepasst werden. Die antibiotische Therapie darf bei noch bestehenden Entzündungszeichen nicht abgesetzt werden, sondern ist bis zur vollständigen Infektkontrolle fortzuführen. Bei Ausbleiben einer adäquaten Wirksamkeit der Antibiotikatherapie sollte frühzeitig ein Mikrobiologe oder Infektiologe in die medikamentöse Behandlung miteinbezogen werden [25].

\section{Rekonstruktive Eingriffe}

Nach vollständiger Sanierung des Infekts sowie kardiozirkulatorischer Stabilisierung des Patienten sollte zügig die Rekonstruktion der Hautweichteildefekte erfolgen, da andernfalls schwere bleibende Funktionseinschränkungen aufgrund von Gelenkeinsteifung oder Sehnenverklebung drohen. Bis zur Rekonstruktion können entweder konsequent befeuchtete Verbände oder auch Unterdrucksysteme zum Einsatz kommen. Allerdings dürfen diese Verbandssysteme keinesfalls zur Induktion von Granulationsgewebe, um z. B. den Wundgrund mit exponierten Sehnen oder Knochen für eine Spalthauttransplantation zu "konditionieren“, verwendet werden. Diese Vorgehensweise führt unweigerlich zu irreversiblen massiven Funktionseinschränkungen und ist vor dem Hintergrund der Verfügbarkeit vielfältiger rekonstruktiver Verfahren obsolet.

Während das initiale Débridement keinesfalls unter Berücksichtigung der später notwendigen Defektrekonstruktion erfolgen darf - hier würde sonst das Risiko für ungünstige chirurgische Kompromisse bestehen - sollte danach schnellstmöglich eine präzise Planung des rekonstruktiven Vorgehens vorgenommen werden. Parallel dazu wird unter analgetischer Abschirmung frühzeitig mit einer vorsichtigen physiotherapeutischen Beübung begonnen.

In Abhängigkeit von der Defektgröße und -tiefe stehen verschiedene Rekonstruktionsmöglichkeiten zur Verfügung. Oberste Priorität hat ein suffizienter und funktionell stabiler Wundverschluss, der eine Kompromittierung tiefer liegender funktioneller Strukturen sicher verhindert. Bei kleineren Befunden mit hautsparenden Resektionen ist manchmal eine Sekundärnaht möglich. In den meisten Fällen ist bei exponiertem Muskelgewebe eine Spalthauttransplantation das Verfahren der Wahl. Bei freiliegenden funktionellen Strukturen wie Knochen, Sehnen oder Nerven besteht die Indikation für eine gestielte oder freie Lappenplastik. Dabei sollten v. a. im Bereich der Beuge- oder Strecksehnen fasziokutane Lappenplastiken bevorzugt werden, da diese im Rahmen von evtl. notwendigen Sekundäreingriffen wie Tenolysen etc. problemlos angehoben werden können und das Risiko postoperativer Adhäsionen geringer ist. Aufgrund des hohen Standardisierungsgrads sind inzwischen mikrochirurgische freie Lappenplastiken auch bei multimorbiden Patienten mit Erfolgsraten von $>95 \%$ umsetzbar. Die Wahl des rekonstruktiven Verfahrens orientiert sich ausschließlich an den funktionellen und ästhetischen Erfordernissen. Gegebenenfalls ist die Verlegung in ein Zentrum mit entsprechender rekonstruktiver Expertise erforderlich.

Amputationen an der oberen Extremität sind aufgrund einer deutlichen Einschränkung der Funktion und Lebensqualität nur bei nichtbeherrschbaren Infektionen oder völlig funktionsloser Extremität durchzuführen. Ins- 

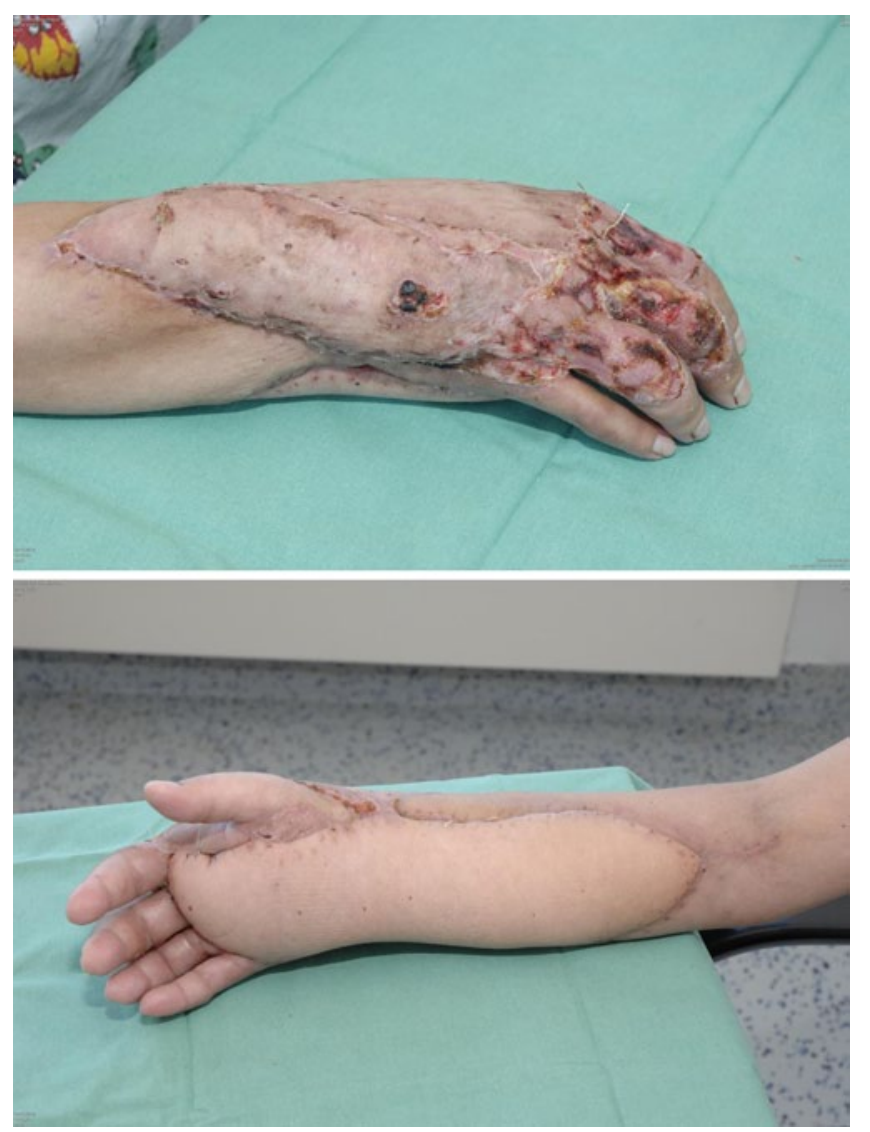

Abb. $3 \Delta$ Klinischer Befund am 23. postoperativen Tag nach Weichteilrekonstruktion mit 2 separaten freien Lappenplastiken (streckseitig: Latissimus-dorsi-Lappenplastik, beugeseitig: Parascapularlappenplastik)
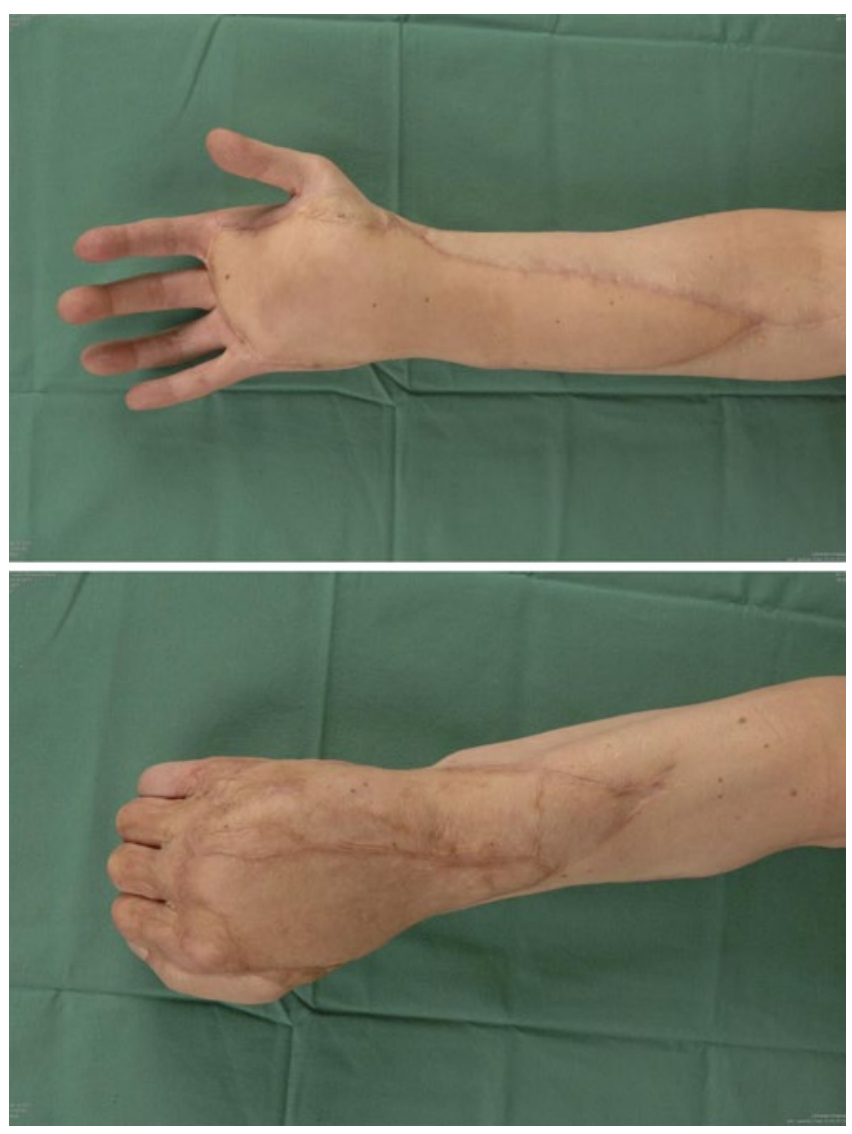

Abb. $4 \Delta$ Klinischer Befund 10 Monate postoperativ besondere bei der NF muss berücksichtigt werden, dass aufgrund der meist ausgedehnten Defekte nach Amputationen die prothetische Versorgung sehr eingeschränkt sein kann.

\section{Rehabilitation}

An die initiale Rekonstruktion schließt sich die Rehabilitation an. Bei ausgedehnten Befunden mit entsprechend großen funktionellen Defiziten und drohender hoher Minderung der Erwerbsfähigkeit (MdE) ist eine fachgerechte Rehabilitation essenziell, da nur so ein zufriedenstellendes funktionelles Ergebnis erzielt werden kann. Hier besteht häufig bei motorischen oder auch kombinierten Defiziten die Indikation zu stationären Heilverfahren. Auch können Sekundäreingriffe wie Arthrolysen, Tenolysen, Sehnenrekonstruktionen und Narbenkorrekturen erforderlich werden (s. $\bullet$ Abb. 1).

\section{Fallbeispiel}

Anamnese. 41-jährige Patientin beklagte neben rezidivierendem Fieber bis $38^{\circ} \mathrm{C}$ eine massive Schwellung und Schmerzen in der rechten Hand sowie im distalen Unterarm. Vorausgegangen war eine Schnittverletzung am rechten Daumen eine Woche zuvor. Keine relevanten Vorerkrankungen bekannt.

Aufnahmebefund. Kreislaufstabil, massive Schwellung der rechten Hohlhand mit beginnenden Spannungsblasen, livide Verfärbungen der Langfinger; Aufnahmelabor: Leukozyten $21,6 \times 10^{9} / 1$, C-reaktives Protein 183,5 mg/l; in der Röntgendiagnostik kein Hinweis auf einen pathologischen Befund.

Verlauf. Es erfolgte die stationäre Aufnahme der Patientin und das notfallmäßige, radikale Débridement sämtlicher entzündlich veränderter Faszien an Unterarm und Hand dorsal und palmar.
Initial wurde kalkuliert eine intravenöse Tripleantibiose mit Penicillin, Clindamycin und Meronem eingeleitet. Weitere 3 Operationen mit Débridements waren notwendig, bis die Weichteilinfektion unter Kontrolle war. Die resultierenden Weichteildefekte wurden durch 2 freie Lappenplastiken gedeckt: Streckseitig erfolgte die Weichteilrekonstruktion mit einer Latissimus-dorsi-Lappenplastik mit Anschluss an die A. radialis sowie an eine dorsale Armvene. Der beugeseitige Defektverschluss gelang durch eine Parascapularlappenplastik mit separatem Anschluss an die A. radialis und an eine Begleitvene. Zudem war eine Spalthauttransplantation erforderlich. Trotz vitaler Bedrohung in der Infektsituation konnte durch eine radikale operative Sanierung eine Amputation an der oberen Extremität verhindert werden. Anschließend gelang mittels mikrochirurgischer Rekonstruktion ein stabiler Defektverschluss. Zur Verbesserung der motorischen Funktion sowie des Er- 
scheinungsbildes erfolgten eine Tenolyse der Strecksehnen der Hand sowie eine beidseitige Lappenausdünnung (• Abb. 2, 3,4).

Mikrobiologie. Nachgewiesen wurden $\beta$-hämolysierende Streptokokken der Gruppe A, Proteus mirabilis sowie Staphylococcus aureus.

Histopathologie. Es zeigten sich abszedierende und phlegmonöse Weichgewebeentzündungen mit Nekrosenbildung, insgesamt vereinbar mit dem Bild einer nekrotisierenden Fasziitis.

Epikrise. 45 Tage stationärer Aufenthalt, 11 Tage intensivmedizinische Behandlung, 6 Operationen.

\section{Fazit für die Praxis}

\section{- Die nekrotisierende Fasziitis stellt ein lebensbedrohliches Krankheits- bild dar. \\ - Die Diagnose wird anhand klinischer Symptome und der Anamnese ge- stellt. \\ - Bei Verdacht auf NF ist die notfall- mäßige chirurgische Exploration immer Therapie der Wahl. \\ - Für den Behandlungserfolg ist ein radikales chirurgisches Vorgehen ent- scheidend. \\ - Häufig sind mehrere Operationen notwendig, um die Infektionsherde vollständig zu sanieren. \\ - Eine breite systemische Antibiotika- therapie ist obligat. \\ - Zur Rekonstruktion der Weich- teildefekte sind Spalthauttrans- plantation sowie gestielte oder freie Lappenplastiken möglich. \\ - Die frühzeitige Verlegung in ein Schwerbrandverletztenzentrum kann sinnvoll sein.}

\section{Korrespondenzadresse}

\section{Dr. med. M.S.T. Wähmann}

Klinik für Hand-, Plastische- und Rekonstruktive Chirurgie - Schwerbrandverletztenzentrum, BG Klinik Ludwigshafen, Lehrkrankenhaus der Ruprecht-Karls-Universität Heidelberg Ludwig-Guttmann-Str. 13, 67071 Ludwigshafen matthias.waehmann@bgu-ludwigshafen.de

\section{Literatur}

1. Bilton B, Zibari G, McMillan R, Aultman D et al (1998) Aggressive surgical management of necrotizing fasciitis serves to decrease mortality: a retrospective study. Am Surg 64:397-400

2. Boyer A, Vargas F, Coste F, Saubusse E, Castaing Y, Gbikpi-Benissan G et al (2009) Influence of surgical treatment timing on mortality from necrotizing soft tissue infections requiring intensive care management. Intensive Care Med 35(5):847-853

3. Elliott DC, Kufera JA, Myers RA (1996) Necrotizing soft tissue infections. Risk factors for mortality and strategies for management. Ann Surg 224(5):672683

4. Elliott D, Kufera JA, Myers RA (2000) The microbiology of necrotizing soft tissue infections. Am J Surg 179(5):361-366

5. Faucher LD et al (2001) Burn center management of necrotizing soft-tissue surgical infections in unburned patients. Am J Surg 182(6):563-569

6. Fournier JA (1988) Jean-Alfred Fournier 18321914. Gangrene foudroyante de la verge (overwhelming gangrene). Sem Med 1883. Dis Colon Rectum 31(12):984-988

7. Gonzalez MH (1998) Necrotizing fasciitis and gangrene of the upper extremity. Hand clin 14(4):635-645

8. Green RJ, Dafoe DC, Raffin TA (1996) Necrotizing fasciitis. Chest 110(1):219-229

9. Hankins CL, Southern S (2008) Factors that affect the clinical course of group A beta-haemolytic streptococcal infections of the hand and upper extremity: a retrospective study. Scand J Plast Reconstr Surg Hand Surg 42(3):153-157

10. Haywood CT, McGeer A, Low DE (1999) Clinical experience with 20 cases of group A streptococcus necrotizing fasciitis and myonecrosis: 19951997. Plast Reconstr Surg 103:1567-1573

11. Herr M, Grabein B, Palm HG et al (2011) Nekrotisierende Fasziitis. Update 2011. Unfallchirurg 114:197-216

12. Hohendorff B, Seyler S, Naik M, Biber F, Franke J (2015) Amputation der Hand bei nekrotisierender Fasziitis: Ein Fallbericht. Handchir Mikrochir Plast Chir 47(1):62-66

13. Jones J (1871) Surgical memories of the war of the rebellion: investigation upon the nature, causes and treatment of hospital gangrene as prevailed in the federate armies. US Sanitary Commission, New York

14. Kaul R, McGeer A, Low DE, Green K, Schwartz B (1997) Population-based surveillance for group $A$ streptococcal necrotizing fasciitis: clinical features, prognostic Indicators, and microbiologic analysis of seventy-seven cases. Ontario Group A Streptococcal Study. Am J Med 103(1):18-24

15. Levy MM, Dellinger RP, Townsend SR, LindeZwirble WT, Marshall JC, Bion J et al (2010) The Surviving Sepsis Campaign: results of an international guideline-based performance improvement program targeting severe sepsis. Intensive Care Med 36(2):222-231

16. Lim YJ, Yong FC, Wong $\mathrm{CH}$, Tan AB (2006) Necrotising fasciitis and traditional medical therapy- a dangerous liaison. Ann Acad Med Singapore 35(4):270-273

17. Lopez FA, Lartchenko S (2006) Skin and soft tissue infections. Infect Dis Clin North Am 20(4):759-772, v-vi. doi:10.1016/j.idc.2006.09.006

18. McHenry CR et al (1995) Determinants of mortality for necrotizing soft-tissue infections. Ann Surg 221(5):558-563
19. Osterman M, Draeger R, Stern P (2014) Acute hand infections. J Hand Surg 39(8):1628-1635

20. Puvanendran R, Huey JC, Pasupathy S (2009) Necrotizing fasciitis. Can Fam Physician 55(10):981-987

21. Roldan CJ (2008) Necrotizing fasciitis. J Emerg Med 34(4):457-458. doi:10.1016/j. jemermed.2007.07.016

22. Rybak MJ et al (2009) Vancomycin therapeutic guidelines: a summary of consensus recommendations from the Infectious Diseases Society of America, the American Society of Health-System Pharmacists, and the Society of Infectious Diseases Pharmacists. Clin Infect Dis 49(3):325-327

23. Ryssel H, Germann G, Kloeters O, Radu CA, Reichenberger M, Gazyakan E (2010) Necrotizing fasciitis of the extremities: 34 cases at a single centre over the past 5 years. Arch Orthop Trauma Surg 130(12):1515-1522

24. Ryssel H, Germann G, Riedel K et al (2007) Chirurgisches Konzept und Er- gebnisse bei nekrotisierender Fasziitis. Chirurg 78:1123-1129

25. Schmid MR, Kossmann T, Duewell S (1998) Differentiation of necrotizing fasciitis and cellulitis using MR imaging. AJR Am J Roentgenol 170(3):615-620

26. Schnurer S, Beier JP, Croner R, Rieker RJ, Horch RE (2012) [Pathogenesis, classification and diagnosis of necrotizing soft tissue infections]. Chirurg 83(11):943-952

27. Sudarsky LA, Laschinger JC, Coppa GF, Spencer FC (1987) Improved results from a standardized approach in treating patients with necrotizing fasciitis. Ann Surg 206(5):661-665

28. Sunderland IR, Friedrich JB (2009) Predictors of mortality and limb loss in necrotizing soft tissue infections of the upper extremity. J Hand Surg Am 34(10) 1900-1901

29. Talan DA, Moran GJ, Abrahamian FM (2008) Severe sepsis and septic shock in the emergency department. Infect Dis Clin North Am 22(1):1-31

30. Taviloglu K, Yanar H (2007) Necrotizing fasciitis: strategies for diagnosis and management. World J Emerg Surg 2:19

31. Ustin JS, Malangoni MA (2011) Necrotizing softtissue infections. Crit Care Med 39(9):2156-2162

32. Wilson B (1952) Necrotizing fasciitis. Am Surg 18:416

33. Wong $\mathrm{CH}$, Wang YS (2005) The diagnosis of necrotizing fasciitis. Curr Opin Infect Dis 18(2):101106 